\title{
Comparación entre la revascularización mediante cirugía abierta y angioplastia infrainguinal en isquemia crítica de miembros inferiores en el Hospital Central de Chihuahua
}

\section{Comparison between revascularization through open surgery and infrainguinal angioplasty in patients with critical limb ischemia in the Central hospital of Chihuahua}

\author{
Karen Fernández-Díaz* y Omar Hernández-Hurtado \\ Departamento de Angiología y Cirugía Vascular, Hospital Central del Estado de Chihuahua, Chihuahua, México
}

\begin{abstract}
Resumen
Objetivo: Comparar los resultados entre la revascularización abierta y la angioplastia infrainguinal como medidas terapéuticas para salvar las extremidades inferiores en pacientes con isquemia crítica en el Hospital Central de Chihuahua, México. Material y métodos: Estudio observacional de cohorte retrospectiva. Se incluyeron los pacientes sometidos a revascularización por isquemia crítica en el periodo de marzo del 2016 a marzo del 2019. La estratificación fue de acuerdo a la modalidad de tratamiento y clasificación de Rutherford. Evaluamos el salvamento de la extremidad a 1 año, supervivencia libre de amputación y mortalidad perioperatoria en todos los pacientes. Resultados: Identificamos 72 pacientes: 33 (45.8\%) con bypass infrainguinal, $38(52.7 \%)$ con angioplastia percutánea y 1 (1.3\%) con manejo híbrido. El seguimiento a 1 año después de la revascularización muestra similar salvamento de extremidades y supervivencia libre de amputación en ambos grupos de revascularización, con un salvamento del $66.7 \%$ en los pacientes sometidos a bypass y del $73.7 \%$ en el grupo de angioplastia, con un total de salvamento del $70.8 \%(p=0.65)$. No hay una diferencia estadísticamente significativa entre ambas opciones terapéuticas. Conclusión: En el presente estudio no hay diferencias significativas en la mortalidad o salvamento de la extremidad entre ambos tipos de revascularización. La decisión clínica seguirá estando limitada por las opciones de revascularización basadas en el tipo de lesiones arteriales y las características propias del paciente.
\end{abstract}

Palabras clave: Isquemia crítica. Cirugía de bypass. Angioplastia percutánea. Salvamento de extremidad. Supervivencia libre de amputación.

\section{Abstract}

Objective: To compare the results between open revascularization and infrainguinal angioplasty as therapeutic measures in the salvage of the lower extremities in patients with critical ischemia at the Central Hospital of Chihuahua, Mexico. Material and methods: Retrospective cohort observational study. Patients under revascularization for critical ischemia during the period from March 2016 to March 2019 were included. The stratification was according to the selection of treatment

Correspondencia:

*Karen Fernández Díaz

E-mail: kimerstix@hotmail.com bajo la licencia CC BY-NC-ND (http://creativecommons.org/licenses/by-nc-nd/4.0/).
Fecha de recepción: 26-09-2019 Fecha de aceptación: 21-10-2019
DOI: 10.24875/RMA.19000004
Disponible en internet: 10-12-2019 Rev Mex Angiol. 2019;47(4):30-35 www.RMAngiologia.com 
and classification of Rutherford. Evaluation of limb salvage at 1 year, amputation-free survival and perioperative mortality in all patients. Results: We identified 72 patients, 33 (45.8\%) infrainguinal bypass, 38 (52.7\%) percutaneous angioplasty and $1(1.3 \%)$ hybrid management. Follow-up at 1 year after revascularization, shows similar limb salvage and amputation-free survival in both revascularization groups; Patients under bypass with a salvage of $66.7 \%$, while the angioplasty group was $73.7 \%$, with a total salvage of $70.8 \%, p=0.65$, there is no statistically significant difference between these therapeutic options. Conclusion: In the present study there are no differences in mortality or limb salvage between both types of revascularization. The clinical decision included is limited by revascularization options based on the type of arterial lesions and the patient's own characteristics.

Key words: Critical ischemia. Bypass surgery. Percutaneous angioplasty. Limb salvage. Amputation-free survival.

\section{Introducción}

La enfermedad arterial periférica (EAP) se debe al bloqueo de las arterias que suministran sangre a las extremidades inferiores, generalmente secundaria a aterosclerosis. La manifestación clínica más grave es la isquemia crítica de las extremidades $(\mathrm{IC})^{1}$, estado de insuficiencia arterial manifestado por perfusión tisular crónica inadecuada en reposo². Factores de riesgo independientes asociados con la progresión a IC incluyen fumar, diabetes, edad avanzada y disfunción renal crónica $^{3}$. La prevalencia de IC es del $1 \%$ de la población adulta. Entre el 5 y el $10 \%$ de los pacientes con EAP asintomática 0 claudicación intermitente progresará a IC durante un período de 5 años $^{3}$. En ausencia de revascularización exitosa, el 20 a $40 \%$ de los pacientes requerirán amputación, y más del $20 \%$ muere a los 6 meses $^{4}$. Los criterios para el diagnóstico incluyen: 1) más de 2 semanas de dolor en reposo recurrente en el pie que requiere el uso regular de analgésicos y es asociado con una presión sistólica del tobillo de $\leq 50 \mathrm{mmHg}$ o una presión sistólica del dedo del pie de $\leq 30 \mathrm{mmHg} 02$ ) herida que no cicatriza o gangrena del pie 0 dedos de los pies ${ }^{5}$.

La elección del examen diagnóstico debe determinarse con un enfoque individualizado de la evaluación anatómica para cada paciente, incluido el riesgo-beneficio ${ }^{6}$.

La revascularización es la terapia primaria para la IC, la terapia médica sirve como agente terapéutico esencial adjunto. Ayuda a acelerar la curación de heridas, prevenir la amputación, y mejorar la calidad de vida, incluye el uso de antiplaquetarios, bloqueadores $\beta$, estatinas, inhibidores de la enzima convertidora de angiotensina y modificación de factores de riesgo, como dejar de fumar ${ }^{7-9}$.

Las amputaciones debidas a isquemia crítica son frecuentes, a pesar del avance en las técnicas de revascularización ${ }^{10}$. Un número importante de pacientes requieren amputación contralateral $(5.7 \%$ y $11.5 \%$ en el primer y quinto año, respectivamente, después de la primera amputación), tienen úlceras isquémicas recurrencias 0 mueren ${ }^{9,11}$. Cada intervención debe realizarse para ofrecer el más eficiente, seguro y oportuno tratamiento de revascularización rentable para los pacientes ${ }^{12}$.

Actualmente, hay una tendencia significativa hacia el «enfoque endovascular primero», teniendo en cuenta la mayor invasividad, riesgo de morbimortalidad asociada con la revascularización quirúrgica y los avances significativos tecnológicos en el área endovascular7. La revascularización quirúrgica debe ser preferida en pacientes con IC que son relativamente activos, más jóvenes, tienen una esperanza de vida $>2$ años y pueden soportar los rigores de una cirugía abierta ${ }^{13,14}$. La terapia endovascular incluye la angioplastia con balones simples o con liberación de fármacos, aterectomía y uso de estents desnudos o cubiertos, y se asocia con una disminución de la morbilidad y la mortalidad periprocedimiento ${ }^{15}$. La decisión de recomendar la revascularización quirúrgica o endovascular varía de acuerdo a diferentes factores del paciente (patrón de enfermedad, disponibilidad de conductos autógenos y comorbilidades), factores externos (capacitación médica y habilidades quirúrgicas y endovasculares) y acceso a un procedimiento ${ }^{15}$.

Cuando un procedimiento ofrece mejoría en el dolor, cicatrización de la herida y conservación de la extremidad debe ser tomada en cuenta como una opción real y efectiva para ser utilizada ${ }^{16}$.

\section{Material y métodos}

Estudio observacional retrospectivo, con información obtenida del expediente clínico y los censos de pacientes ingresados en el Servicio de Angiología y Cirugía Vascular del Hospital Central de Chihuahua durante el periodo de marzo de 2016 a marzo de 2019. Al tratarse de un estudio retrospectivo, de consulta de registros de cirugías y expedientes clínicos, se garantizó la confidencialidad y anonimato de los pacientes en particular y de acuerdo a los códigos internacionales de ética de 
Tabla 1. Características de los pacientes y comorbilidades

\begin{tabular}{|l|c|c|c|}
\hline & Bypass (n = 38) & Angioplastia (n=33) & Híbrido (n = 1) \\
\hline $\begin{array}{l}\text { Género } \\
\text { - Hombres } \\
- \text { Mujeres }\end{array}$ & $25(34.7 \%)$ & $19(26.38 \%)$ & $1(1.38 \%)$ \\
\hline Edad & $17(23.6 \%)$ & $10(13.8 \%)$ & 58 \\
\hline Rutherford IV & 66.5 & 69.8 & $1(1.38 \%)$ \\
\hline Rutherford V & $10(13.8 \%)$ & $12(16.6 \%)$ & $1(2.17 \%)$ \\
\hline Rutherford VI & $23(31.9 \%)$ & $20(27.7 \%)$ & $1(1.58 \%)$ \\
\hline Tabaquismo & $2(2.7 \%)$ & $4(5.5 \%)$ & 0 \\
\hline Diabetes mellitus & $20(43.4 \%)$ & $25(54.3 \%)$ & $32(50.795)$ \\
\hline HAS & $30(47.61 \%)$ & $21(39.6 \%)$ & $2(25 \%)$ \\
\hline Insuficiencia renal & $32(60.3 \%)$ & & \\
\hline
\end{tabular}

la investigación. No fue requerida la aprobación del comité de ética, y fue autorizado por el Comité Local de Investigación.

Los datos se obtuvieron de la revisión manual de los expedientes clínicos tanto físicos como digitales y de los registros quirúrgicos del Servicio de Cirugía vascular, comprendidos durante el periodo ya comentado. En el estudio se incluyeron todos los pacientes con diagnóstico de isquemia crítica sometidos a proceso de revascularización de una extremidad inferior, manejados por el Servicio de Angiología y Cirugía Vascular con el expediente completo, pacientes de cualquier edad y sexo.

De la muestra total de 78 pacientes, 6 pacientes fueron excluidos del estudio por no contar con el expediente completo o por expediente perdido, por lo que se obtuvo una muestra de 72 pacientes. La revisión de cada expediente se concentró en la recolección de los datos demográficos, factores de riesgo, grado de isquemia según la clasificación de Rutherford, índice tobillo-brazo (ITB), sector arterial afectado, cirugía de revascularización realizada (angioplastia o bypass) y se determinó la mortalidad perioperatoria y amputación mayor subsecuente.

El vaciamiento de datos se realizó utilizando el programa Microsoft ${ }^{\circledR}$ Excel para la depuración y manejo de tablas y el software SPSS ${ }^{\circledR}$ (IBMTM Statistical Package for the Social Science versión 22.0) (SPSS, Chicago, IL, EE. UU.) para el análisis de la información. Como parte del análisis estadístico, se utilizó chi cuadrada para proporciones y para las variables nominales y categóricas; mientras que para las variables continuas utilizamos la prueba t de Student para muestras independientes a una significancia de 0.05 .

\section{Resultados}

Durante el periodo de 36 meses fueron evaluados 72 pacientes de forma retrospectiva, de los cuales 45 eran hombres $(62.5 \%)$ y 27 mujeres (37.5\%), con un promedio de edad de 68.9 años (en un rango de 41 a 90 años). La tabla 1 muestra las características y comorbilidades de los pacientes.

En relación con el ITB, se observó que, en el grupo en el que se logró salvar de la extremidad, el promedio fue de 0.52, mientras que en el grupo en el que se presentó pérdida de la extremidad, el promedio fue de $0.49(p=0.62)$.

Se obtuvo mayor salvamento de la extremidad en pacientes no fumadores, con un $73.1 \%$, contra el $69.6 \%$ en el grupo de fumadores $(p=0.75)$.

En cuanto al diagnóstico de base, en los pacientes con EAP no diabéticos, la extremidad se salvó en el $88.9 \%$, en comparación con el $68.3 \%$ en el grupo de diabéticos $(p=0.20)$. Presentaron mayor salvamento de la extremidad a 1 año los pacientes con escala de Rutherford IV, con un $91.3 \%$, en el grupo con escala de Rutherfod V fue del $61.9 \%$, mientras que en el grupo de escala de Rutherford VI fue del $66.7 \%(p=0.033)$. La correlación arteriográfica entre los sectores arteriales muestra que el territorio más afectado fue el de los vasos tibiales, en un $51.39 \%$, seguido del sector femoropoplíteo, en un $43.06 \%$, y ambos sectores en un $5.56 \%$.

El seguimiento a 1 año después de la cirugía de bypass y angioplastia percutánea en pacientes con isquemia crítica de extremidades muestra similar salvamento de extremidades y supervivencia libre de 
amputación en ambos grupos de revascularización. Los pacientes sometidos a bypass tuvieron un salvamento de la extremidad del $66.7 \%$, mientras que en el grupo de angioplastia fue del 73.7 , y del $100 \%$ para la cirugía híbrida, siendo el total de salvamento de la extremidad del $70.8 \%(p=0.65)$, por lo tanto, si nos basamos en estos resultados, no hay diferencia estadísticamente significativa entre ambas opciones terapéuticas (Tabla 2).

La amputación mayor a 1 año de acuerdo al tipo de procedimiento de revascularización en los pacientes sometidos a bypass fue del $33 \%$, mientras que en el grupo de angioplastia fue del $26.3 \%$, con un total de $29.2 \%(p=0.65)$ (Tabla 3).

En cuanto a mortalidad perioperatoria por tipo de procedimiento de revascularización, se observó que los pacientes sometidos a bypass tuvieron una mortalidad del $3 \%$, mientras que en el grupo de angioplastia fue del $2.8 \%$, con una mortalidad total del $2.9 \%$ $(p=0.98)$ (Tabla 4).

\section{Discusión}

Los pacientes con IC tienen un alto riesgo de pérdida de extremidades sin revascularización y un alto riesgo a corto plazo de eventos cardiovasculares. La revascularización está indicada si previene la pérdida de la extremidad y preserva la ambulación y la función.

La amputación no es la opción terapéutica, aunque a corto plazo parezca la opción más resolutiva, a largo plazo conlleva un aumento de la morbimortalidad y un impacto en los servicios de salud.

El bypass y la angioplastia percutánea son los métodos estándar de tratamiento, cada uno de ellos presenta ventajas, indicaciones, retos técnicos y complicaciones específicas.

A pesar de que hay estudios clínicos, como el ensayo prospectivo $\mathrm{BASIL}^{17}$, y una serie de estudios observacionales ${ }^{18}$, todavía es incierto cuál de los métodos de tratamiento debe utilizarse en un paciente específico ${ }^{19}$.

En el estudio BASIL, publicado hace más de 10 años, la colocación de un estent endovascular no fue permitida en el grupo de angioplastia. La introducción posterior de los nuevos dispositivos de aterectomía, balones recubiertos de medicamento, estents de nitinol y estents liberadores de fármacos tiene el potencial de mejorar la durabilidad y los resultados clínicos a largo plazo en pacientes tratados con terapia endovascular.

La revascularización endovascular ofrece un riesgo inicial más bajo que la cirugía abierta, pero la enfermedad recurrente por reestenosis o la enfermedad de novo es común en pacientes con IC. Los resultados
Tabla 2. Salvamento de extremidad estratificado por procedimiento de revascularización

\begin{tabular}{|l|c|c|c|c|}
\hline \multirow{2}{*}{} & \multicolumn{2}{|c|}{$\begin{array}{c}\text { Salvamento de } \\
\text { extremidad n (\%) }\end{array}$} & \multirow{2}{*}{ Total } & \\
\cline { 2 - 3 } & Salvamento & Pérdida & & \\
\hline $\begin{array}{l}\text { Procedimiento de } \\
\text { revascularización }\end{array}$ & & & & \\
$\begin{array}{l}\text { Bypass } \\
\text { Angioplastia }\end{array}$ & $22(66.7)$ & $11(33.3)$ & $33(100)$ & 0.65 \\
\hline Híbrido & $28(73.7)$ & $10(26.3)$ & $38(100)$ & \\
\hline Total & $1(100)$ & 0 & $1(100)$ & \\
\hline
\end{tabular}

*Prueba chi cuadrada para proporciones.

Tabla 3. Amputación mayor estratificada por procedimiento de revascularización

\begin{tabular}{|l|c|c|c|c|}
\hline & \multicolumn{2}{|c|}{ Amputación mayor n (\%) } & \multirow{2}{*}{ Total } & \multirow{2}{*}{ p } \\
\cline { 2 - 3 } & Sí & No & & \\
\hline $\begin{array}{l}\text { Procedimiento de } \\
\text { revascularización }\end{array}$ & & & \\
\hline $\begin{array}{l}\text { Bypass } \\
\text { Angioplastia }\end{array}$ & $11(33)$ & $22(66.7)$ & $33(100)$ & 0.65 \\
\hline Híbrido & $0(26.3)$ & $28(73.7)$ & $38(100)$ & \\
\hline Total & $21(29.2)$ & $51(70.8)$ & $72(100)$ & \\
\hline
\end{tabular}

*Prueba chi cuadrado para proporciones.

Tabla 4. Mortalidad perioperatoria estratificada por procedimiento de revascularización

\begin{tabular}{|c|c|c|c|c|}
\hline & \multicolumn{2}{|c|}{ Mortalidad a 30 días n (\%) } & \multirow[t]{2}{*}{ Total } & \multirow[t]{2}{*}{$\mathbf{p}^{*}$} \\
\hline & Finado & Sobrevida & & \\
\hline $\begin{array}{l}\text { Procedimiento de } \\
\text { revascularización } \\
\text { Bypass } \\
\text { Angioplastia } \\
\text { Híbrido }\end{array}$ & $\begin{array}{c}1(3) \\
1(2.8) \\
0(0)\end{array}$ & $\begin{array}{c}32(97) \\
35(97.2) \\
1(100)\end{array}$ & $\begin{array}{c}33(100) \\
36(100) \\
1(100)\end{array}$ & 0.98 \\
\hline Total & $2(2.9)$ & 68 (97.1) & $70(100)$ & \\
\hline
\end{tabular}

*Prueba chi cuadrada para proporciones.

del ensayo BASIL demostraron que, después de 2 años, las dos estrategias no tuvieron una diferencia significativa en la supervivencia libre de amputación. No hubo diferencia en la calidad de vida relacionada con la salud entre las dos estrategias, pero para el primer año, los costos hospitalarios asociados con la cirugía fueron alrededor de un tercio más altos que los 
relacionados con la angioplastia, y hubo menos complicaciones con el procedimiento endovascular. A los 4 años, la cirugía de bypass con injerto de vena tiene mejor salvamento de las extremidades que la angioplastia percutánea. Algunos estudios informan de resultados a 5 años ${ }^{20}$, pero esto $s$ son inconsistentes y las comparaciones son difíciles debido a diferencias en las modalidades de tratamiento.

En el presente estudio, los resultados obtenidos para el objetivo general, que era el seguimiento a 1 año después de la cirugía de bypass y de la angioplastia percutánea, y donde la elección del método de tratamiento se basó en la experiencia local en materia de éxito técnico y características de las lesiones y del paciente a tratar, muestran similar salvamento de extremidades y supervivencia libre de amputación en ambos grupos de revascularización. Los pacientes sometidos a bypass tuvieron un salvamento del $66.7 \%$, mientras que el grupo de angioplastia fue del $73.7 \%$, siendo en total de $70.8 \%$ ( $p=0.65)$, por tanto, no hay diferencia estadísticamente significativa entre ambas opciones terapéuticas. En relación con los objetivos específicos, se debe resaltar que la necesidad de amputación mayor subsecuente fue más elevada en el grupo de los pacientes sometidos a bypass, con un $33 \%$, mientras que en el grupo de angioplastia fue del $26.3 \%$. Ambos grupos son semejantes, lo que refuerza la idea de que no hay diferencia estadísticamente significativa entre ambas técnicas de revascularización. En cuanto al análisis de mortalidad perioperatoria por tipo de procedimiento de revascularización, se observó que los pacientes sometidos a bypass tuvieron una mortalidad del $3 \%$, mientras que en el grupo de angioplastia fue del $2.8 \%$.

Un factor trascendental en el tratamiento de la isquemia crítica es al momento del diagnóstico, pues este influye en la progresión de la mala perfusión y pérdida irreversible de tejido, lo que limita las opciones terapéuticas. Encontramos que los pacientes con escala IV de Rutherford, el $91.3 \%$ presentó salvamento de la extremidad.

El ITB ha quedado limitado al diagnóstico de la enfermedad arterial periférica, ya que se han realizado estudios clínicos, donde se realiza seguimiento de revascularización basados en la modificación del ITB, sin encontrarse relación directa o modificación significativa del mismo. En relación con el ITB, el promedio en el grupo donde se logró el salvamento de la extremidad fue de 0.52 , mientras que en el grupo donde se presentó pérdida de la extremidad, el promedio fue de 0.49. Debe considerarse el uso de ITB como medida cuantitativa de la enfermedad, en especial como parámetro en el seguimiento o respuesta a la revascularización.

La enfermedad multinivel involucró tanto el sector femoropoplíteo como el sector infrapoplíteo, lo que respalda lo mencionado por otros autores en relación con la fisiopatología, gravedad y progresión de la enfermedad aterosclerótica; el sector arterial más afectado fue el de vasos tibiales, en un $51.39 \%$, seguido del sector femoropoplíteo, en un $43.06 \%$, y ambos sectores, en un $5.56 \%$.

El presente trabajo de investigación tiene como limitante principal el número de casos involucrados, sin embargo, es la muestra con la que se cuenta en el Servicio de Angiología y Cirugía vascular del Hospital Central de Chihuahua, que es un centro de referencia estatal. Esto influye en el análisis estadístico y, consecuentemente, en los resultados, es decir, el que no exista diferencia estadísticamente significativa pero sí clínica.

\section{Conclusiones}

En el presente estudio no parece haber diferencias significativas en la mortalidad o salvamento de las extremidades entre la revascularización endovascular y quirúrgica en pacientes con IC. Sin embargo, cabe destacar que los pacientes con diabetes mellitus tuvieron un peor pronóstico en cuanto a salvamento de la extremidad en comparación con el grupo de pacientes con EAP. Estos datos respaldados en la literatura internacional derivan de un estudio clínico aleatorizado y muchos estudios observacionales, y la presencia de heterogeneidad clínica de estos resultados nos da conclusiones inciertas y destaca la necesidad de una mayor investigación.

La decisión clínica para pacientes con IC seguirá estando limitada por las opciones de revascularización basadas en el tipo de lesiones arteriales, características propias del paciente, preferencia y juicio clínico.

\section{Conflicto de intereses}

Los autores declaran que no existe conflicto de intereses alguno.

\section{Responsabilidades éticas}

Protección de personas y animales. Los autores declaran que para esta investigación no se han realizado experimentos en seres humanos ni en animales. 
Confidencialidad de los datos. Los autores declaran que en este artículo no aparecen datos de pacientes. Derecho a la privacidad y consentimiento informado. Los autores declaran que en este artículo no aparecen datos de pacientes.

\section{Bibliografía}

1. Krishna SM, Moxon JV, Golledge J. A Review of the Pathophysiology and Potential Biomarkers for Peripheral Artery Disease. Int J Mol Sci. 2015; 11294-322

2. Lookstein RA, Ikeno F, Geraghty P, Vranckx P, Ho M, Rundback J, et al. Evaluation and Treatment of Patients With Lower Extremity Periphera Artery Disease. J Am Coll Cardiol. 2015;65(9):931-41.

3. Farber A, Eberhardt RT. The Current State of Critical Limb Ischemia: A Systematic Review. JAMA Surg. 2019:151(11):1070-77.

4. Menard MT, Farber A, Assmann SF, Choudhry NK, Conte MS Creager MA, et al. Design and Rationale of the Best Endovascular Versus Best Surgical herapy for Patients With Critical Limb Ischemia (BESTCLI) Trial.. J Am Heart Assoc. 2016;5(7). pii: e003219.

5. Armstrong EJ, Alam S, Henao S, Lee AC, Derubertis BG, Montero-baker M, et al. Multidisciplinary Care for Critical Limb Ischemia: Current Gaps and Opportunities for Improvement. J Endovasc Ther. 2019;26(2):199-212.

6. Gerhard-Herman MD, Gornik HL, Barrett C, Barshes NR, Corriere MA Drachman DE. 2016 AHA/ACC Guideline on the Management of Patients With Lower Extremity Peripheral Artery Disease: Executive Summary: A Report of the American College of Cardiology/American Heart Association Task Force on Clinical Practice Guidelines. J Am Coll Cardiol. 2017;69(11):1465-508.

7. Elsayed S, Clavijo LC. Critical limb ischemia. Cardiol Clin. 2015;33(1):37-47.

8. Chung J, Timaran DA, Modrall JG, Ahn C, Timaran CH, Kirkwood ML, et al. Optimal medical therapy predicts amputation-free survival in chronic critical limb ischemia. J Vasc Surg. 2013;58(4):972-80.
9. Goodney PP, Travis LL, Nallamothu BK, Holman K, Suckow B, Henke PK, et al. Variation in the use of lower extremity vascular procedures for critical limb ischemia. Circ Cardiovasc Interv. 2013;5(01):94-102.

10. Rodriguez T, Escoto $S$. Cirugía de Salvamento de miembros inferiores con isquemia crítica. Rev Mex Angiol. 2008;36(3):88-97.

11. Glaser JD, Bensley RP, Hurks R, Dahlberg S, Hamdan AD, Wyers MC et al. Fate of the contralateral limb after lower extremity amputation. J Vasc Surg. 2013;58(06): 1571-7.e1.

12. Durazzo TS, Frencher S GR. Influence of race on the management of lower extremity ischemia: revascularization vs amputation. JAMA Surg. 2013;148(07):617-23.

13. Conte MS. Critical appraisal of surgical revascularization for critical limb ischemia. J Vasc Surg. 2013:57(2, Suppl):8S-13S.

14. Kabra A, Suresh K, Vivekanand V et al. Outcomes of angiosome and non-angiosome targeted revascularization in critical lower limb ischemia. J Vasc Surg. 2013;57(1):44-9.

15. Conte MS. Bypass versus Angioplasty in Severe Ischaemia of the Leg (BASIL) and the (hoped for) dawn of evidence-based treatment for advanced limb ischemia. J Vasc Surg. 2010;51(5 Suppl.):69S-75S

16. Bradbury AW, Adam DJ, Beard JD, Cleveland T, Forbes JF, Fowkes FGR, et al. Bypass versus angioplasty in severe ischaemia of the leg (BASIL): Multicentre, randomised controlled trial. Lancet. 2005;366(9501):1925-34.

17. Lecuona $\mathrm{H}$, Rodriguez $\mathrm{T}$. Angioplastia percutánea infrapoplítea en pacientes con isquemia crítica en el Servicio de Angiología, Cirugía Vascular y Endovascular. CMN 20 de Noviembre, ISSSTE. Rev Mex Angiol. 2013;41(4):188-208

18. Altreuther M, Mattsson E. Long-Term Limb Salvage and Amputation-Free Survival After Femoropopliteal Bypass and Femoropopliteal PTA for Critical Ischemia in a Clinical Cohort. 2019;53(7006):112-7.

19. Heidenfelder B, Vemulapalli S, Subherwal S, Patel MR, Dolor RJ, Jones WS, et al. Comparative effectiveness of endovascular and surgical revascularization for patients with peripheral artery disease and critical limb ischemia. Am Heart J. 2014;167(4):489-498.e7.

20. Dosluoglu HH, Lall $P$, Harris LM, Dryjski ML. Long-term limb salvage and survival after endovascular and open revascularization for critical limb ischemia after adoption of endovascular-first approach by vascular surgeons. J Vasc Surg. 2012;56(2):361-71 